\begin{tabular}{|l|l|l|}
\hline \multicolumn{2}{|c|}{ PublisherInfo } \\
\hline \hline PublisherName & $:$ & BioMed Central \\
\hline \hline PublisherLocation & $:$ & London \\
\hline \hline PublisherImprintName & $:$ & BioMed Central \\
\hline \hline
\end{tabular}

\title{
Phase III trial of chemotherapy ? HerceptinR in metastatic breast cancer
}

\begin{tabular}{|l|l|l||}
\hline \multicolumn{2}{|c||}{ ArticleInfo } \\
\hline \hline ArticleID & $:$ & 3766 \\
\hline \hline ArticleDOI & $:$ & $10.1186 /$ bcr-2001-68448 \\
\hline \hline ArticleCitationID & $:$ & 68448 \\
\hline \hline ArticleSequenceNumber & $:$ & 38 \\
\hline \hline ArticleCategory & $:$ & Paper Report \\
\hline \hline ArticleFirstPage & $:$ & 1 \\
\hline \hline ArticleLastPage & $:$ & 3 \\
\hline \hline & & RegistrationDate : 2001-8-20 \\
\hline ArticleHistory & $:$ & Received \\
& $:$ Accepted $2001-4-27$ \\
\hline ArticleCopyright & $:$ & Biomed Central Ltd2001 \\
\hline \hline \hline ArticleGrants & $:$ & \\
\hline \hline ArticleContext & $:$ & 1305833-8-20 \\
\hline \hline
\end{tabular}


Fatima Cardoso, Affl

Aff1 Jules Bordet Institute, Brussels, Belgium

Keywords

Breast cancer, Herceptin, HER2, phase III trial

\section{Context}

Despite advances in diagnosis and treatment, breast cancer still remains a major cause of cancer deaths. Metastatic breast cancer (MBC) is virtually incurable, with median survival of 18 to 24 months. The HER2 gene encodes a growth factor membrane receptor that is overexpressed in $25-30 \%$ of $\mathrm{MBC}$ cases and is associated with aggressive metastasis and poor prognosis. Transtuzumab (HerceptinR) is a humanised monoclonal antibody against HER2 that has proven efficacy as a single-agent therapeutic for MBC. This randomised phase III trial analysed the effect of adding transtuzumab to standard first-line chemotherapy (CT) for MBC.

\section{Significant findings}

In an intention-to-treat analysis, the addition of transtuzumab was associated with statistically significant and better results for time to disease progression ( 7.4 months versus 4.6 months; $P<0.001$ ), response rate $(50 \%$ versus $32 \% ; P<0.001)$, duration of response $(9.1$ months versus 6.1 months; $P$ $<0.001)$ and overall survival $(25.1$ months versus 20.3 months; $P=0.046)$. Similar differences were seen in both chemotherapy subgroups (anthracycline-based and paclitaxel), although for overall survival the $P$ values were 0.16 and 0.17 , respectively. Addition of transtuzumab was also associated with higher cardiotoxicity than that for CT alone, particularly in the anthracycline-based subgroup and in patients who had received adjuvant anthracyclines. After a median follow-up of 30 months the authors concluded that transtuzumab increases the clinical benefit of first-line CT in MBC and reduces the relative risk of death by $20 \%$. 


\section{Comments}

The magnitude of transtuzumab's effect on patient survival in this study may have been reduced by the crossover of patients from randomised to open-label treatment. This change in regimen occurred for $66 \%$ of patients, whose cancer had progressed and thus required treatment with transtuzumab.

Furthermore, patients eligible for this study included those with tumours with different levels $(2+$ and $3+$ of HER 2 overexpression. A better selection of patients, namely only HER $23+$ by IHC or HER 2 2+ by FISH could have allowed a more accurate assessment of transtuzumab benefit. Cardiac dysfunction is an important and troubling side effect of transtuzumab treatment, particularly in patients who have had, or who receive, concomitant anthracycline-based CT; in these patients the cardiotoxicity of anthracyclinebased agents is already a concern. This study confirms transtuzumab as one of the few anticancer agents associated with an important survival benefit in MBC. Establishing its role in the adjuvant setting is essential. Clinical trials are already ongoing in the US, and will soon begin in Europe.

\section{Methods}

Immunohistochemical IHC analysis of HER2 positivity, randomised phase III clinical trial, intentionto-treat analysis, Kaplan-Meier and two-sided log-rank tests

\section{Additional information}

\section{References}

1. Slamon DJ, Leyland-Jones B, Shak S, Fuchs H, Paton V, Bajamonde A, Fleming T, Eirmann W, Wolter J, Pegram M, Baselga J, Norton L: Use of chemotherapy plus a monoclonal antibody against HER2 for metastatic breast cancer that overexpresses HER2. New Eng1 J Med . 2001, 344: 783-792. 